\title{
Structural Design of a Bionic Anti-Clogging Drip Irrigation Emitter Based on Shark Dorsal Fin
}

\author{
Caixiang Wei, Zhengying Wei*, Xueli Chen, Kun He \\ The State Key Laboratory for Manufacturing Systems Engineering, Xi'an Jiaotong University, Xi'an, China \\ Email: *cxwei96@163.com
}

How to cite this paper: Wei, C. X., Wei, Z. Y., Chen, X. L., \& He, K. (2021). Structural Design of a Bionic Anti-Clogging Drip Irrigation Emitter Based on Shark Dorsal Fin. Journal of Geoscience and Environment Protection, 9, 11-20.

https://doi.org/10.4236/gep.2021.94002

Received: March 2, 2021

Accepted: April 9, 2021

Published: April 12, 2021

\begin{abstract}
Due to the poor anti-clogging performance of the common drip irrigation emitters, this paper designed a new bionic flow channel in the emitter based on the shape of shark dorsal fin. After preliminary structural design, the computational fluid dynamics (CFD) simulation showed that the bionic emitter exhibited superior anti-clogging performance and reasonable hydraulic performance. The passage rate of particles of the bionic emitter in simulation reached $96.3 \%$ which was $37.6 \%$ higher than $70 \%$ of traditional emitter, and the discharge exponent reached 0.4995 which was close to traditional emitter. Physical experiments were consistent with the CFD results, which confirmed the correctness of simulation. After a short cycle anti-clogging performance experiment, the bionic emitter still maintained $96.09 \%$ of the initial flow rate.
\end{abstract}

\section{Keywords}

Bionic Drip Irrigation Emitter, Shark Dorsal Fin Flow Channel, Realizable k- $\varepsilon$ Model, Anti-Clogging Performance

\section{Introduction}

A drip irrigation emitter is the terminal of drip irrigation system, which consumes the energy of water flowing so that water flows into the soil in the form of water drops. The common labyrinth channel emitters are widely used because they make water flow into a turbulent flow and have good energy dissipation performance by using complex and changeable boundary (Yu, 2011). However, due to the large sediment content of rivers in most water-scarce regions and sediment deposition under long-term use, drip irrigation emitters are frequently clogged as shown in Figure 1, which becomes a vital factor restricting the development of drip irrigation technology. Therefore, in recent years the research 
direction of emitters mainly focuses on the anti-clogging performance of emitters.

In order to cope with clogging of emitters, Zhou et al. 2019 formed a local vortex by using appropriate internal water shear force to clean the inner wall of the channel, so as to alleviate the clogging of the emitters. Ait-Mouheb et al. 2018 studied the effects of hydraulic conditions on the deposition of clay particles and biofilm formation by optical method for better comprehension of the physical and biological clogging during drip irrigation, and used a transparent microfluidic system composed of labyrinth channels to identify the most easily clogged areas. Li et al. 2006 used variable geometric parameters to design and construct emitters, and tested the relationship between anti-clogging performance and parameters. So far, the research on the anti-clogging performance of emitters was mostly based on the traditional labyrinth channel. Actually, the channel structure itself has a great impact on the anti-clogging performance, so it may be possible to find a new channel structure to solve this problem.

Bionic design is not only an important research method in industry, but also a significant means of innovation, and profiling is a common bionic method in product design (Luo et al., 2018). Shark dorsal fin (Figure 2(a)), with a flat front tip, whose height and thickness gradually increase backwards along the spine, makes sharks swim steadily. The fluid resistance of the dorsal fin is less in the course of swimming because of its streamline on the vertical plane and sharp

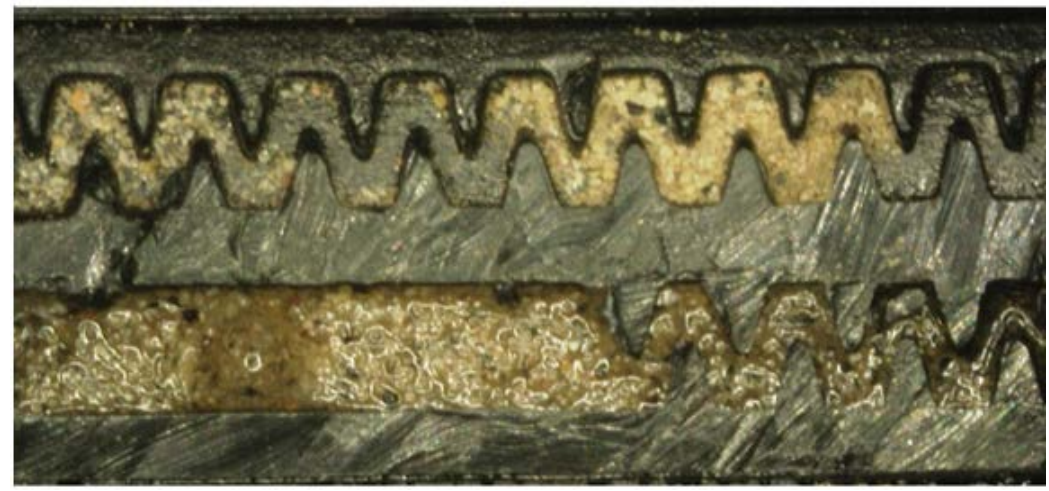

Figure 1. Clogging of emitters in practical application.

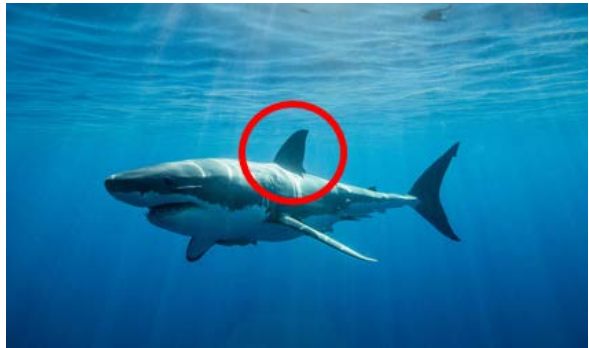

(a)

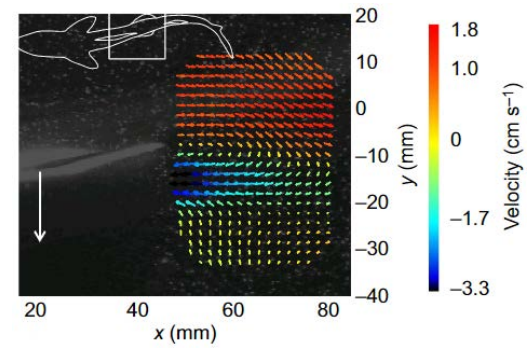

(b)

Figure 2. Shark dorsal fin and the velocity behind the dorsal fin (Maia et al. 2017). 
triangle on the horizontal plane. From the perspective of fluid dynamics, this structure can weaken the shark's thickening of the surface layer during swimming, reduce the viscous resistance, and inhibit the tail flow separation. Maia et al. 2017 observed the flow field near the shark dorsal fin by time-resolved particle image velocimetry (Figure 2(b)), and found that the fluid would accelerate when passing through the sides of the dorsal fin, which could make water flow more capable of carrying sand particles in irrigation emitters. Besides, the antenna on the top of the vehicle mostly adopts the shape of the shark dorsal fin, which can effectively guide the air flow and make the movement of the fluid smoother (Zhang et al., 2017; Demasi et al., 2016; Sudo et al., 2008). According to this, this paper designed a bionic channel structure based on the streamlined shark dorsal fin and hoped that particles can flow out of the emitter with water, in order to improve anti-clogging performance of the emitter.

\section{Materials and Methods}

\subsection{Physical Model}

The structural parameters of shark dorsal fin were extracted and simplified for bionic design. The 3-D model of the bionic emitter with inlet and outlet was shown in Figure 3, with filter grids at the entrance. For each unit, the overall structure was symmetrically designed, with the shape of shark dorsal fin in the middle, so that the fluid carrying particles flowed steadily and rapidly through both sides of the dorsal fin. The outer sides of the channel were parallel to the sides of the dorsal fin. The rear part of the unit was arc-shaped, preventing particle deposition and consuming turbulent kinetic energy to limit flow rate. The length of each unit was $2.25 \mathrm{~mm}$, width $2 \mathrm{~mm}$, depth $1 \mathrm{~mm}$, and there were 22 units in total. The parametric design was carried out, and four structural parameters were selected. Figure 4 shows the structure and four feature parameters of the bionic emitter channel unit, where $W_{1}$ is the width of top rear fin, $W_{2}$ is main channel width, $L$ is the length of top fin and $\alpha$ is the angle of anterior fin.

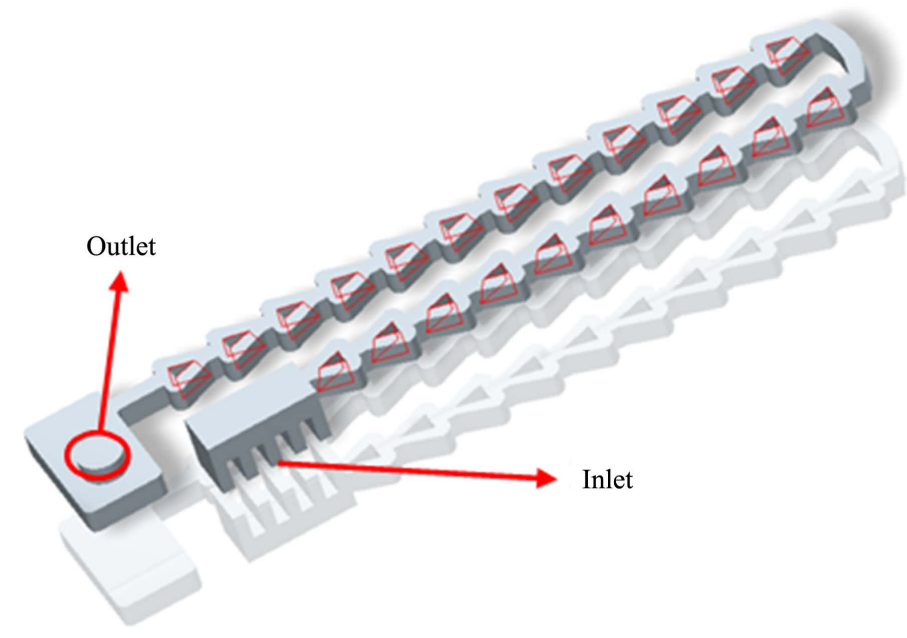

Figure 3. 3-D model of the fluid in flow channel. 


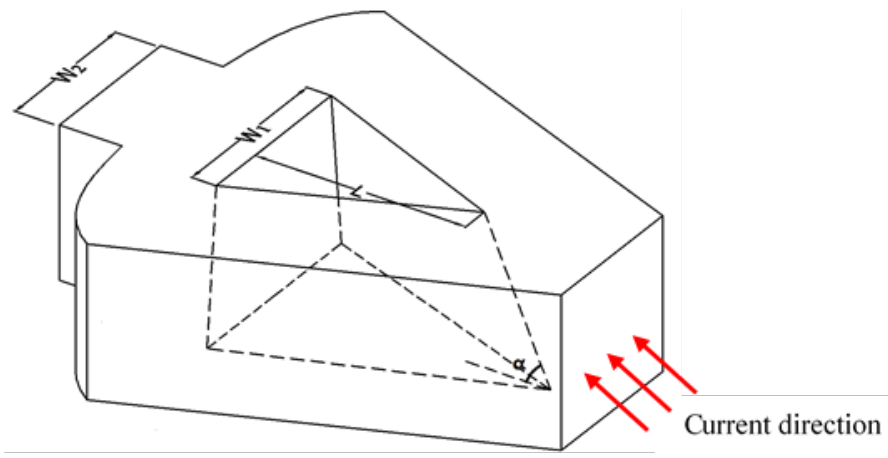

Figure 4. Parametric design of the bionic emitter unit.

\subsection{Numerical Methods}

The water flow inside the flow channel conformed to the mass conservation and momentum conservation theorem. So the following assumptions were made about the flow: normal temperature, steady flow, neglect of the mass force and surface tension of the fluid, viscous incompressible fluid and no consideration about the effect of the surface roughness of the flow channel. The standard k- $\varepsilon$ turbulence model and realizable $\mathrm{k}-\varepsilon$ turbulence model met the requirements. Shaheed et al. 2019 pointed out the better performance of the standard k- $\varepsilon$ model in curved channels and the realizable $\mathrm{k}-\varepsilon$ model in confluent channels. Since the structure designed in this paper had confluent channels, the realizable $\mathrm{k}-\varepsilon$ model was chosen.

Realizable k- $\varepsilon$ model (Lateb et al., 2013) includes a new realizable eddy viscosity and a new model dissipation rate formula. The constant $\mathrm{C}_{\mu}$ of turbulent eddy viscosity achieves the realizable effect. It is no longer a constant but a function of the turbulent fields, average strain and rotation rate. The model has been widely validated for various flows including boundary layer flows and separated flows. Transport equation for $k$ and $\varepsilon$ in this model:

$$
\begin{gathered}
\frac{\partial k}{\partial t}+\mathrm{U}_{j} \frac{\partial k}{\partial x_{j}}=\frac{\partial}{\partial x_{j}}\left(\frac{v_{t}}{\sigma_{k}} \frac{\partial k}{\partial x_{j}}\right)-\overline{\mathrm{u}_{i} \mathrm{u}_{j}} \mathrm{U}_{i, j}-\varepsilon \\
\frac{\partial \varepsilon}{\partial t}+\mathrm{U}_{j} \frac{\partial \varepsilon}{\partial x_{j}}=\frac{\partial}{\partial x_{j}}\left(\frac{v_{t}}{\sigma_{\varepsilon}} \frac{\partial \varepsilon}{\partial x_{j}}\right)+\mathrm{C}_{1} S \varepsilon-\mathrm{C}_{2} \frac{\varepsilon^{2}}{k+\sqrt{v \varepsilon}}
\end{gathered}
$$

where $\varepsilon$ is the turbulent dissipation rate, $k$ the turbulent kinetic energy, $\sigma_{\varepsilon}$ the dissipation with the value of $1.2, v$ the kinematic viscosity, $v_{t}$ the turbulent viscosity. $\mathrm{C}_{1}=\max [0.43, \eta /(\eta+5)]$ with $\eta=\frac{k}{S} S$, where $S$ is defined by the velocity strain rate tensor, $\mathrm{C}_{2}=1.9$. The Reynolds stress term $\overline{\mathrm{u}_{i} \mathrm{u}_{j}}$ represents the momentum diffusion transport by turbulent motion.

The standard wall function method was adopted near the wall surface, and finite volume method (Mousavian \& Najafl, 2009) was selected as discrete method. The type of inlet was pressure-inlet which was set to $30-100 \mathrm{kPa}$, with an interval of $10 \mathrm{kPa}, 8$ levels in total. The gauge pressure of outlet was atmospheric pressure $(0 \mathrm{kPa})$. Pressure-velocity coupling method used the SIMPLE algo- 
rithm. Spatial discretization terms were solved by the second order upwind method, and convergent residual accuracy was set to 0.0001 .

A discrete term was created under Fluent model option. The fluid was treated as the continuous phase, the sand of $2500 \mathrm{~km} / \mathrm{m}^{3}$ density was treated as the discrete phase, and injection type was surface injection. The diameter of particles was within $0.06 \mathrm{~mm}$ to $0.18 \mathrm{~mm}$, with the average size of $0.12 \mathrm{~mm}$. Discrete random walk model (Euler-Lagrange two-phase flow model) was checked under turbulent dispersion option, which had the advantages: 1) no pseudo-diffusion in numerical calculation, 2) no more excessive calculation memory for disperse particles, 3) the complex process of particle collisions can be easily added (Sabnis et al., 1992). The number of tries was set to 5 , which meant that the same number of particles was released 5 times and the latter release had an impact on the former, which was more consistent with the actual situation (Zhang et al., 2020). Tracking length scale was a value of $0.1 \mathrm{~mm}$.

\subsection{Physical Test for Model Validation}

Hydraulic performance tests were carried out by 3D printing test pieces (Figure 5) to verify the consistency between the numerical simulation and the physical results. The inner diameter of the test piece was $16 \mathrm{~mm}$, material was photosensitive resin and the manufacturing error was less than $0.1 \mathrm{~mm}$. The part of the flow channel was sealed with plastic perforated at the outlet. The pressure was applied to the pipeline on the test platform by water pump, and the precise pressure gauge was installed at the inlet and outlet of the pipeline to ensure the accuracy of the water pressure. The flow rate of the emitter was measured by measuring cylinder. Before measuring the flow rate, the pipeline valve should be adjusted so that the pressure indicator reached the pressure level and remained unchanged. After 3 minutes waiting, the mass flow rate within 5 minutes was measured (Yang et al., 2020). The above steps were repeated to obtain the flow rate at each pressure level. This study carried out two hydraulic performance tests, one at the initial design stage and the other after optimization. There were 25 bionic emitters installed on the test platform, and the average value was taken as the result.

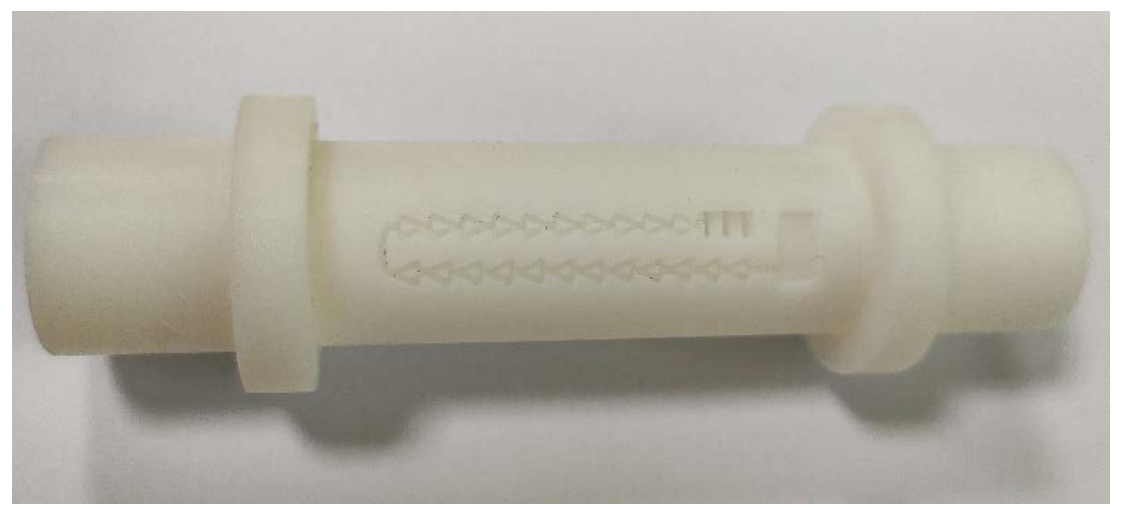

Figure 5. Test sample of the bionic emitter. 
Anti-clogging performance test referred to "Clogging test methods for emitters" drafted by ISO committee which suggested that the emitter was considered to be clogged when the flow rate of the emitter was $75 \%$ less than the initial flow rate, that was, the flow rate was reduced by more than $25 \%$. The inert particles were natural river sand and the working pressure of the inlet was $50 \mathrm{kPa}$. The test consisted of eight stages of adding sand. Clear water flowed stably in 5 drip irrigation belts composed of 25 emitters and the average of the flow rate of each emitter within 5 min was measured as reference before the test. Subsequently the larger size particle was added continuously with the progress of each stage and fully mixed with irrigation water until eight stages were accomplished. The system required running for 15 minutes under the condition of stable pressure before each measure, and preparation between the end of the previous stage and the beginning of the next stage needed to be carried out under no pressure.

\subsection{Evaluation Indicators of Emitters}

The evaluation of emitters generally has two indicators, one is hydraulic performance, and the other is anti-clogging performance. Hydraulic performance (Keller \& Karmeli, 1974) is determined by discharge exponent $x$ from the following:

$$
\mathrm{Q}=\mathrm{k} \cdot \mathrm{p}^{\mathrm{x}}
$$

where $\mathrm{Q}$ is the flow rate $(\mathrm{L} / \mathrm{h})$, kthe discharge coefficient, $\mathrm{p}$ the working pressure indicated by head $(\mathrm{m}) . x$ reveals the sensitivity of the flow rate to the change of pressure. The greater the $x$ is, the more sensitive and the worse hydraulic performance. In simulation anti-clogging performance is determined by passage rate of particles $\eta$ which is the ratio of particles passing through the outlet $\eta_{p}$ to particles entering the inlet $\eta_{t}$ (Zhang et al., 2011).

$$
\eta=\frac{\eta_{p}}{\eta_{t}}
$$

Hydraulic performance and anti-clogging performance restrict each other, so an efficient multi-objective algorithm is needed to find a balance between the two performances.

\section{Results}

\subsection{Turbulence Characteristics of Flow Channel}

Figure 6 shows the velocity distribution in partial flow channel obtained by numerical simulation at the $50 \mathrm{kPa}$ pressure inlet. There are two high-speed mainstreams on both sides of the fin in each unit, which carries water flow and particles passing quickly. Due to the high external flow velocity and low pressure in the area behind the fin, two vortices are formed here, which not only is one of the main energy dissipation forms of the fluid in the flow channel, but also has a certain scouring effect on the wall surface. The vortex area in the flow channel is a dynamic area, which transforms continuously with the disturbance of water flow and has no dead angle, thus sand particles are not easily deposited here 


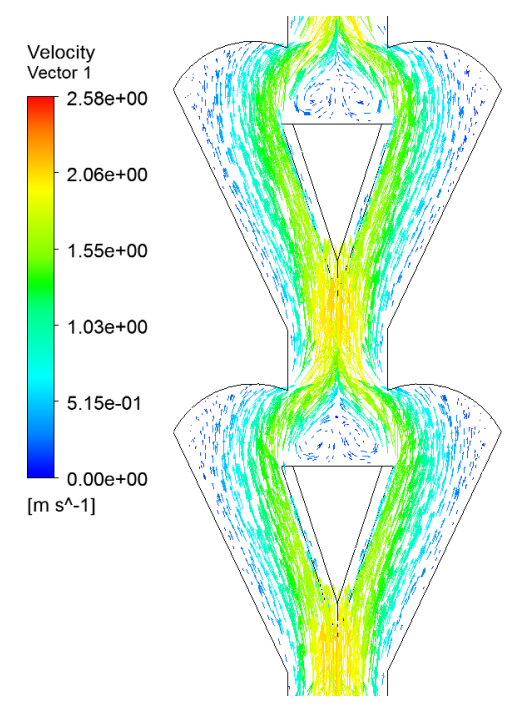

Figure 6. Velocity vector in the flow channel.

(Vieira et al., 2007; Adin \& Sacks, 1991). The rear arc-shaped structure greatly changes the direction of the two mainstreams so that they recombine in the opposite directions when they flow out of the unit, which helps to consume the energy of the water flow to ensure the hydraulic performance of the bionic emitter.

\subsection{Particle Tracks of Discrete Phase}

The irregular path line in Figure 7 is the track of a single particle in the emitter, and the black outline is the middle section of the wall surface. The time required for the particle to pass through each unit is close and extremely short, which is a good illustration of the rapid passage of the particle in the flow channel with water flow without falling into a vortex, retention and sedimentation. The particle passes through each unit nearby the dorsal fin with the mainstream. Although the particle hits the arc-shaped structure at the rear in some units, it still bounces back to the mainstream quickly. Finally, the particles escape from the flow channel after about $0.13 \mathrm{~s}$ at the outlet.

\subsection{Comparison between Numerical Simulation and Physical Validation}

The pressure-discharg curves obtained from the hydraulic performance experiment of the bionic emitter is shown in Figure 8. The discharge exponent $x$ is 0.4995 , corresponding to structure parameters $W_{1}=0.6, W_{2}=0.65, L=0.9$ and $\alpha=75^{\circ}$. The relative error between experiment and simulation is small, the maximum does not exceed $4.36 \%$.

Through two-phase flow simulation, the passage rate of particles $\eta$ is $97.36 \%$. However, the physical experiment cannot directly verify the correctness of the passage rate of particles, but there is a corresponding relationship between $\eta$ and the flow reduction rate of anti-clogging experiment. The higher the passage rate of particles is, the lower the reduction rate is. Figure 9 shows the result of 
particle-tracks-1
Particle Residence Time

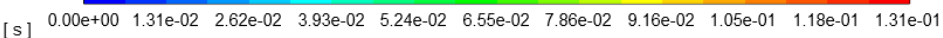

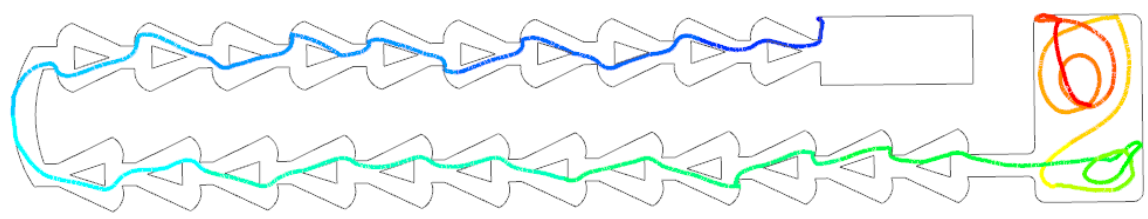

Figure 7. Track of a single particle.

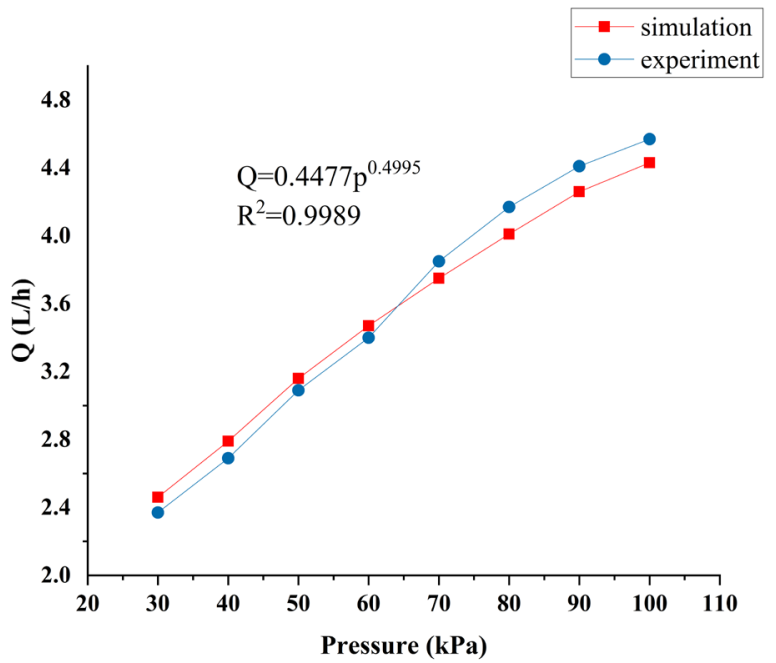

Figure 8. Pressure-discharge rate curves of simulation and the hydraulic experiment.

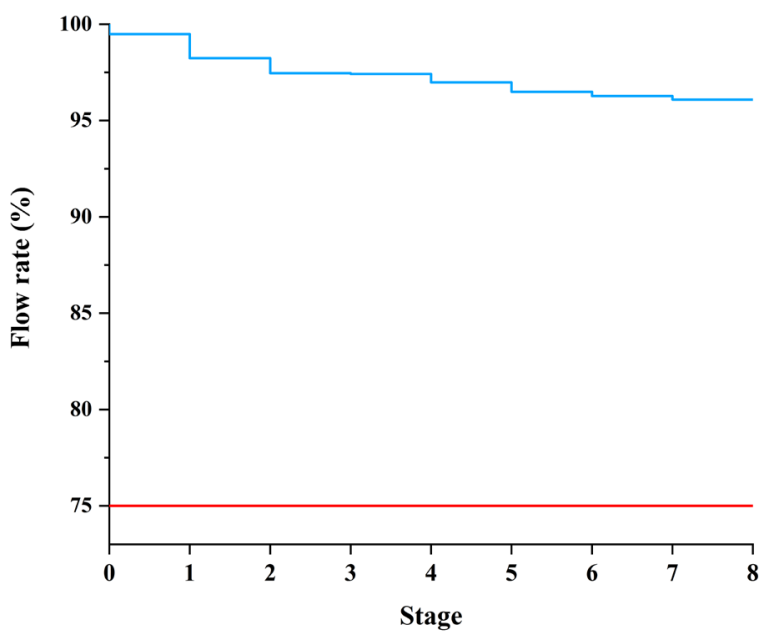

Figure 9. Results of short cycle anti-clogging test.

"short cycle anti-clogging test" at $50 \mathrm{kPa}$ operate pressure as mentioned in 2.3, and $75 \%$ of the initial flow rate is the critical value. As can be seen from the figure that the flow rate has hardly any decreased until the eighth stage decreased by $3.91 \%$ which was also far below the critical value of $25 \%$. Therefore, the physical experiment and simulation of the anti-clogging performance of the emitter have also reached agreement. 
We also did simulations on some popular dentiform channel emitters and found that their discharge exponents $x$ s were between 0.48 and 0.5 which were equivalent to the bionic emitter designed in this paper, and the average passage rate of particles reached $70 \%$ which is much lower than $97.36 \%$ of the bionic emitter. The corresponding flow rates of the shark dorsal fin channel structure under various working pressures are higher than traditional emitters, so that the bionic emitter is suitable for low pressure conditions ( 40 - $70 \mathrm{kPa}$ ) which simultaneously reduces energy consumption.

\section{Conclusion}

In this paper, the bionic emitter with the flow channel designed by imitating the shape of shark dorsal fin is suitable for use in water-scarce areas with large sediment content and in the case of low pressure or large water demand of crops. The superior anti-clogging performance and reasonable hydraulic performance of the emitter were settled by numerical simulations and verified by physical experiments.

\section{Acknowledgements}

This study was supported by the National Key Research and Development Plans of China (2016YFC04010202) and (2017YFD0201504-2). We would like to express our heartfelt thanks to those who helped this research and the reviewers who would help improve this manuscript.

\section{Conflicts of Interest}

The authors declare no conflicts of interest regarding the publication of this paper.

\section{References}

Adin, A., \& Sacks, M. (1991). Dripper-Clogging Factors in Wastewater Irrigation. Journal of Irrigation and Drainage Engineering, 117, 813-826. https://doi.org/10.1061/(ASCE)0733-9437(1991)117:6(813)

Ait-Mouheb, N., Schillings, J., \& Al-Muhammad, J. (2018). Impact of Hydrodynamics on Clay Particle Deposition and Biofilm Development in a Labyrinth-Channel Dripper. Irrigation Science, 37, 1-10. https://doi.org/10.1007/s00271-018-0595-7

Demasi, L., Santarpia, E., Dipace, A., Cavallaro, R., \& Gordnier, R. E. (2016). Aerodynamic and Structural Studies of a Flapping Wing in Forward Flight. AIAA Journal, 1-14. https://doi.org/10.2514/1.J054496

Keller, J., \& Karmeli, D. (1974). Trickle Irrigation Design Parameters. Transaction ASAE, 17, 678-684. https://doi.org/10.13031/2013.36936

Lateb, M., Masson, C., Stathopoulos, T., \& Bédard, C. (2013). Comparison of Various Types of k- $\varepsilon$ Models for Pollutant Emissions around a Two-Building Configuration. Journal of Wind Engineering and Industrial Aerodynamics 115, 9-21. https://doi.org/10.1016/j.jweia.2013.01.001

Li, G. Y., Wang, J. D., Alam, M., \& Zhao, Y. F. (2006). Influence of Geometrical Parameters of Labyrinth Flow Path of Drip Emitters on Hydraulic and Anti-Clogging Perfor- 
mance. Transactions of the ASABE, 49, 637-643. https://doi.org/10.13031/2013.20483

Luo, S. J., Zhang, Y. F., Bian, Z., \& Chan, P. (2018). Status and Progress of Product Shape Bionic Design. Journal of Mechanical, 54, 138-155. (In Chinese) https://doi.org/10.3901/JME.2018.21.138

Maia, A., Lauder, G. V., \& Wilga, C. D. (2017). Hydrodynamic Function of Dorsal Fins in Spiny Dogfish and Bamboo Sharks during Steady Swimming. Journal of Experimental Biology ,220, 3967-3975. https://doi.org/10.1242/jeb.152215

Mousavian, S. M., \& Najafl, A. F. (2009). Numerical Simulations of Gas-Liquid-Solid Flows in a Hydrocyclone Separator. Archive of Applied Mechanics, 79, 395-409. https://doi.org/10.1007/s00419-008-0237-2

Sabnis, J. S., Jong, F., \& Gibeling, H. (1992). A Two-Phase Restricted Equilibrium Model for Combustion of Metalized Solid Propellants. AIAA Journal. https://doi.org/10.2514/6.1992-3509

Shaheed, R., Mohammadian, A., \& Kheirkhah Gildeh, H. (2019). A Comparison of Standard $\mathrm{k}-\varepsilon$ and Realizable k- $\varepsilon$ Turbulence Models in Curved and Confluent Channels. Environmental Fluid Mechanics, 19, 543-568. https://doi.org/10.1007/s10652-018-9637-1

Sudo, S., Tsuyuki, K., Yano, T., \& Takagi, K. (2008). A Magnetic Fluid Microdevice Using Insect Wings. Journal of Physics Condensed Matter, 20, 204142. https://doi.org/10.1088/0953-8984/20/20/204142

Vieira, L. G. M., Silva, C. A., Damasceno, J. J. R., \& Barrozo, M. A. S. (2007). A Study of the Fluid Dynamic Behaviour of Filtering Hydrocyclones. Separation and Purification Technology, 58, 282-287. https://doi.org/10.1016/j.seppur.2007.05.029

Yang, B., Wang, J., \& Zhang, Y. (2020) Anti-Clogging Performance Optimization for Dentiform Labyrinth Emitters. Irrigation Science, 38, 275-285.

https://doi.org/10.1007/s00271-020-00671-6

Yu, L. M. (2011). Relations between the Hydraulic Performance and the Structural Parameters of Emitter. Journal of Changsha University of Science and Technology (Natural Science), 8, 30-35. (In Chinese)

Zhang, J., Zhao, W., Tang, Y., \& Lu, B. (2011). Structural Optimization of Labyrinth-Channel Emitters Based on Hydraulic and Anti-Clogging Performances. Irrigation Science, 29, 351-357. https://doi.org/10.1007/s00271-010-0242-4

Zhang, W. Q., Niu, W. Q., Li, G. C., Wang, J., \& Wang, Y. B. (2020). Lateral Inner Environment Changes and Effects on Emitter Clogging Risk for Different Irrigation Times. Agricultural Water Management, 233, 106069. https://doi.org/10.1016/j.agwat.2020.106069

Zhang, Y., Pan, Z. Y., Gu, Z. Q., Liu, S. C., \& Song, Y. H. (2017). A Research on Bionic Aerodynamic Drag Reduction of Vehicle Body Based on Shark Fins. Automotive Engineering, 39, 1018-1024+1029. (In Chinese)

Zhou, B., Li, Y., \& Liu, Y. (2019). Critical Controlling Threshold of Internal Water Shear Force of Anti-Clogging Drip Irrigation Emitters Using Reclaimed Water. Irrigation Science, 37, 469-481. https://doi.org/10.1007/s00271-019-00624-8 